\title{
Image quality of low mA CT pulmonary angiography reconstructed with model based iterative reconstruction versus standard CT pulmonary angiography reconstructed with filtered back projection: an equivalency trial
}

\author{
Xavier Montet • Anne-Lise Hachulla • \\ Angeliki Neroladaki • Frederic Lador • Thierry Rochat • \\ Diomidis Botsikas Christoph D. Becker
}

Received: 10 June 2014 / Revised: 3 November 2014 / Accepted: 9 December 2014 / Published online: 21 December 2014

(C) European Society of Radiology 2014

\begin{abstract}
Objective To determine whether CT pulmonary angiography (CTPA) using low mA setting reconstructed with model-based iterative reconstruction (MBIR) is equivalent to routine CTPA reconstructed with filtered back projection (FBP).

Methods This prospective study was approved by the institutional review board and patients provided written informed consent. Eighty-two patients were examined with a low $\mathrm{mA}$ MBIR-CTPA $(100 \mathrm{kV}, 20 \mathrm{~mA})$ and 82 patients with a standard FBP-CTPA (100 kV, $250 \mathrm{~mA})$. Region of interests were drawn in nine pulmonary vessels; signal-to-noise ratio (SNR) and contrast-to-noise ratio (CNR) were calculated. A five-point scale was used to subjectively evaluate the image quality of FBP-CTPA and low mA MBIR-CTPA.

Results Compared to routine FBP-CTPA, low mA MBIRCTPA showed no differences in the attenuation measured in nine pulmonary vessels, higher SNR $(56 \pm 19$ vs $43 \pm 20$, $p<0.0001)$ and higher CNR $(50 \pm 17$ vs $38 \pm 18, p<0.0001)$ despite a dose reduction of $93 \%(p<0.0001)$. The subjective image quality of low mA MBIR-CTPA was quoted as diagnostic in $98 \%$ of the cases for patient with body mass index less than $30 \mathrm{~kg} / \mathrm{m}^{2}$.

Conclusion Low mA MBIR-CTPA is equivalent to routine FBP-CTPA and allows a significant dose reduction while
\end{abstract}

X. Montet $(\bowtie) \cdot$ A.-L. Hachulla $\cdot$ A. Neroladaki · D. Botsikas •

C. D. Becker

Division of Radiology, Geneva University Hospital, Rue

Gabrielle-Perret-Gentil 4, 1211 Geneva 4, Switzerland

e-mail: xavier.montet@hcuge.ch

F. Lador $\cdot$ T. Rochat

Division of Pulmonary Medicine, Geneva University Hospital,

Gabrielle-Perret-Gentil, 4, 1211 Geneva 4, Switzerland improving SNR and CNR in the pulmonary vessels, as compared with routine FBP-CTPA.

Key Points

- Low mA MBIR-CTPA is equivalent to routine FBP-CTPA.

- MBIR-CTPA may be achieved with drastic (93\%) dose reduction.

- Low MA MBIR-CTPA should be studied in the setting of suspected PE.

Keywords Computed tomography pulmonary angiography · Dose $\cdot$ Model-based iterative reconstruction $\cdot$ Signal-to-noise ratio (SNR) · Contrast-to-noise ratio (CNR)

$\begin{array}{ll}\text { Abbreviations } \\ \text { CTPA } & \text { Computed tomography pulmonary angiography } \\ \text { DLP } & \text { Dose-length product } \\ \text { FBP } & \text { Filtered back projection } \\ \text { MBIR } & \text { Model-based iterative reconstruction } \\ \text { SSDE } & \text { Size-specific dose estimate }\end{array}$

Introduction

Pulmonary embolism is a serious medical condition associated with high mortality, ranging from 3 to $6 \%[1,2]$. Computed tomography pulmonary angiography (CTPA) is now widely accepted as the standard test for diagnosing pulmonary embolism (PE) [3], despite its relatively high radiation dose, which varied from 13 to $40 \mathrm{mSv}$ in 2008 [4], but slowly decreased to 3 to $5 \mathrm{mSv}$ nowadays [3].

In order to reduce the radiation dose delivered by CTPA, several strategies have been introduced, including lowering the tube potential $[5,6]$ and lowering the tube current-time 
product $[7,8]$. However, low dose examinations are associated with increased image noise that can now be reduced by newer reconstructions technologies such as iterative reconstructions [9-11].

Model-based iterative reconstruction (MBIR, Veo ${ }^{\circledR}, \mathrm{GE}$ Healthcare, Milwaukee, Wis) is a novel fully iterative [12] reconstruction algorithm allowing reduction of the dose without loss of image quality [13-15]. It has already been demonstrated that chest $\mathrm{CT}$ with an $\mathrm{x}$-ray dose equivalent to a posteroanterior and lateral chest $\mathrm{x}$-ray is possible [16].

The primary aim of this study was to demonstrate equivalency (in terms of signal-to-noise ratio (SNR) in the pulmonary arteries) between standard FBP-CTPA and low $\mathrm{mA}$ (20 mA) MBIR-CTPA. Subjective image quality was also evaluated.

\section{Materials and methods}

This prospective equivalency trial was approved by the institutional review board of the Geneva University Hospital and each patient gave a written informed consent.

\section{Sample size [17]}

Necessary sample size was calculated for SNR as the primary outcome. If there is truly no difference between standard FBPCTPA and low mA (20 mA) MBIR-CTPA, then 138 patients are required in order to be $80 \%$ sure that the limits of a twosided $90 \%$ confidence interval will exclude a difference in SNR means in the main pulmonary artery of more than 10 , assuming a standard deviation of 20 .

\section{Patients}

From January to May 2013, 167 consecutive patients, scheduled for an enhanced chest $\mathrm{CT}$, were examined on a multidetector CT system (Discovery $750 \mathrm{HD}$, GE Healthcare, Milwaukee, Wis). Of these 167 consecutive patients, two patients refused to participate and one changed his mind and asked afterwards to be excluded from the study; thus 164 patients were included in the study. In half of the patients $(n=82)$, a clinical suspicion of PE was the reason for performing CT. These patients were examined with a routine CTPA protocol consisting of intravenous injection of contrast material $(60 \mathrm{ml}$ of Iohexol 350, containing $350 \mathrm{mg} \mathrm{I} / \mathrm{ml}$, GE Healthcare) at a rate of $3.5 \mathrm{ml} / \mathrm{s}$, followed by a $30-\mathrm{ml}$ saline flush at the same flow rate. The patients were asked to hold their breath after mid-inspiration. A region of interest (ROI) was drawn in the main pulmonary artery and imaging started $5 \mathrm{~s}$ after a threshold of $150 \mathrm{HU}$ was reached. The acquisition parameters were as follows: 0.6 -s gantry rotation time, $100 \mathrm{kVp}, 0.984: 1$ beam pitch, 40-mm table feed per gantry rotation, a z-axis tube current modulation was used, with a noise index (NI) of 28 $(\mathrm{min} / \mathrm{max} \mathrm{mA}, 100 / 500)$ and a $64 \times 0.625-\mathrm{mm}$ detector configuration. All routine CTPA were reconstructed with the FBP algorithm (FBP-CTPA).

In the other half of the patients $(n=82)$, the reasons for chest CT were (1) follow-up of a tumour $(n=51)$, (2) search for complication of pneumonia $(n=16)$, (3) follow-up of interstitial pneumonia $(n=11)$, (4) miscellaneous $(n=4)$. In these patients, an enhanced chest $\mathrm{CT}$ was performed $60 \mathrm{~s}$ after contrast media administration ( $60 \mathrm{ml}$ of Iohexol 350, GE Healthcare at a rate of $3.5 \mathrm{ml} / \mathrm{s}$, followed by a $30-\mathrm{ml}$ saline flush at the same flow rate) to answer the clinical questions. A low $\mathrm{mA}(20 \mathrm{~mA})$ dose chest $\mathrm{CT}$ angiography was added for each patient just prior to the enhanced chest $\mathrm{CT}$ (an ROI was placed in the main pulmonary artery, imaging started $5 \mathrm{~s}$ after a threshold of $150 \mathrm{HU}$ was reached). The patients were also asked to hold their breath after mid-inspiration. In order not to increase the dose received by the patient, the dose of the enhanced chest CT was decreased by $10 \%$ by increasing the NI. Modification of the NI changes the $\mathrm{mA}$ (and only the $\mathrm{mA}$ ) used to acquire the image. As previously published, increasing the NI by one unit will result in approximately $10 \%$ decreased dose to the patient [18]. The x-ray dose saved was hence used to acquire the low mA MBIR-CTPA. The low mA MBIR-CTPA protocol was as follow: 0.6-s gantry rotation time, $100 \mathrm{kVp}, 0.984: 1$ beam pitch, 40-mm table feed per gantry rotation, $20 \mathrm{~mA}$ without tube current modulation and a $64 \times 0.625-\mathrm{mm}$ detector configuration. All low mA MBIRCTPA were reconstructed with the model-based iterative reconstruction algorithm.

A known allergy to iodinated contrast media, glomerular filtration rates less than $45 \mathrm{ml} \mathrm{min}{ }^{-1} \mathrm{~m}^{2}$, pregnancy and being younger than 18 years old were considered as exclusion criteria.

Parameters of both protocols, as well as patient demographics, are summarized in Table 1.

Quantitative image analysis

Axial images were reconstructed at $0.625 \mathrm{~mm}$ slice thickness using a soft tissue kernel. All images were evaluated at

Table 1 Protocols and demographic

\begin{tabular}{llll}
\hline & FBP-CTPA & MBIR-CTPA & $p$ \\
\hline $\mathrm{kV}$ & 100 & 100 & 1 \\
$\mathrm{~mA}$, min, max & $100-500$ & 20 & $\mathrm{~N} / \mathrm{A}$ \\
Pitch & $0.984: 1$ & $0.984: 1$ & 1 \\
Gantry rotation time (s) & 0.6 & 0.6 & 1 \\
Noise index & 28 & - & $\mathrm{N} / \mathrm{A}$ \\
Detector configuration & $64 \times 0.625$ & $64 \times 0.625$ & 1 \\
Men/women & $46 / 36$ & $49 / 33$ & 0.64 \\
Age (year) & $64 \pm 15$ & $60 \pm 14$ & 0.08 \\
Body mass index $\left(\mathrm{kg} / \mathrm{m}^{2}\right)$ & $26.1 \pm 6.0$ & $24.8 \pm 4.8$ & 0.14 \\
\hline
\end{tabular}


standard CTPA window settings (window width 350, window level 40).

ROIs were drawn by XM in the (1) main pulmonary artery (MPA), (2) right pulmonary artery (RPA), (3) left pulmonary artery (LPA), (4) right upper lobe artery (RUA), (5) right middle lobe artery (RMA), (6) right lower lobe artery (RLA), (7) left upper lobe artery (LUA), (8) lingular artery (LIA) and (9) left lower lobe artery (LLA). The ROIs were sized to cover almost all the vessel diameters, taking care to avoid artefacts. The mean values (mean attenuation) of these nine ROIs served to calculate the SNR and contrast-to-noise ratio (CNR). Three ROIs were also drawn in each side of the patient (outside the patient) as well as on the anterior part of the patient to assess background noise. The ROIs were sized around $3 \mathrm{~cm}^{2}$ to cover as much air as possible, without including external objects surrounding the patient. The mean values of the three measurements served as background noise (mean background noise). ROIs $\left(2 \mathrm{~cm}^{2}\right)$ were also drawn in the paraspinal and subscapular muscle. The mean values of the two muscles served for contrast-to-noise calculation (mean muscle attenuation):

$\mathrm{SNR}=$ mean attenuation/mean background noise

$\mathrm{CNR}=$ (mean attenuation - mean muscle attenuation $) /$ mean background noise

CT dose index volume (CTDIvol) and dose length product (DLP) were recorded for each examination.

The calculation of the effective dose $(E)$ was based on the "European Guidelines on quality criteria on computed tomography" using the following formula: $E=E_{\mathrm{DPL}} \times \mathrm{DLP}$, where $E$ is the effective dose in $\mathrm{mSv}$ and $E_{\mathrm{DPL}}$ the dose length coefficient for the chest, which is equal to 0.017 [19].

The size-specific dose estimate (SSDE) was also calculated following the recommendation of American Association of Physicists in Medicine (AAPM) report n²04. Briefly, the anteroposterior (AP) and the lateral diameter of each patient were measured at the level of the tracheal bifurcation and summed. The sum of the lateral and AP dimension allows one to find a conversion factor (in the AAPM report $\mathrm{n}^{\circ} 204$, based on a 32-cm-diameter PMMA phantom), which is used to multiply the CTDIvol reported by the scanner to obtain the SSDE [20, 21].

Subjective image quality

All the images were evaluated independently by two radiologists (XM with 10 years and ALH with 7 years of postfellowship experience). Images were anonymised, aggregated in folders in a random way and evaluated in a standard CTPA windows setting (window width 350, window level 40). A five-point scale [5] was used to assess the overall image quality, defined as follows: 1, good image quality at the level of the main pulmonary artery (MPA); 2, good image quality at the level of the pulmonary arteries (RPA and LPA); 3, good image quality at the level of the lobar arteries; 4, good image quality at the level of the segmental arteries; 5, good image quality at the level of the subsegmental arteries.

Good image quality was defined as good visual homogeneous enhancement, without respiratory artefacts, allowing one to confidently exclude PE. In case readers did not reach a score of 5, they were asked to specify why image quality was insufficient to confidently exclude PE at a given level. The cause may be breathing artefacts, insufficient attenuation in the pulmonary vessels due to either bad timing of injection or a too high noise level.

Scores 1-3 were considered insufficient for diagnostic purposes, whereas scores of 4-5 were considered sufficient.

Such a score was used because there is still controversy in the literature as to whether subsegmental pulmonary emboli should be treated or not [3].

Ten cases of routine CTPA, not included in the study, were used to train the readers on image quality.

Statistical analysis

Statistical analysis was performed with Prism (Prism, version 6b, 2012; GraphPad Software, San Diego, CA, USA). For continuous values, the results are presented as mean \pm standard error.

Non-normally distributed datasets (established from Kolmogorov-Smirnov tests) were compared using Friedman test with Dunn post hoc test. Normally distributed data sets were compared using ANOVA test with Bonferroni post hoc test. Two-sided testing was used. Differences were considered significant at $p<0.05$.

\section{Results}

Patient demographics

The FBP-CTPA group comprised 46 men and 36 women with a mean age of $64 \pm 15$ years. The low $\mathrm{mA}$ MBIR-CTPA group comprised 49 men and 33 women with a mean age of $60 \pm$ 14 years (Table 1$)$.

\section{Dose estimates}

The low mA MBIR-CTPA had a significantly lower CTDIvol $(0.59 \pm 0.003$ vs $8 \pm 1.8)$ and DLP $(21 \pm 2$ vs $282 \pm 64)$ than the routine FBP-CTPA protocols (Table 2). Estimated effective doses were $0.3 \pm 0.03$ vs $4.1 \pm 1.1 \mathrm{mSv}, p<0.0001$ (Table 2), whereas SSDE were $0.74 \pm 0.14$ vs $13.3 \pm 3.7 \mathrm{mGy}$ 
Table 2 Comparison of low mA MBIR-CTPA and routine FBP-CTPA

\begin{tabular}{|c|c|c|c|}
\hline & $\begin{array}{l}\text { FBP-CTPA } \\
(n=82) \\
\text { Mean SD }\end{array}$ & $\begin{array}{l}\text { MBIR-CTPA } \\
(n=82) \\
\text { Mean SD }\end{array}$ & $p$ \\
\hline Pulmonary Arteries (HU) & $356 \pm 84$ & $340 \pm 80$ & 0.22 \\
\hline MPA (HU) & $376 \pm 93$ & $361 \pm 81$ & 0.26 \\
\hline RPA (HU) & $368 \pm 88$ & $349 \pm 82$ & 0.16 \\
\hline LPA (HU) & $364 \pm 85$ & $342 \pm 78$ & 0.09 \\
\hline RUA (HU) & $366 \pm 96$ & $356 \pm 95$ & 0.52 \\
\hline RMA (HU) & $339 \pm 86$ & $331 \pm 79$ & 0.56 \\
\hline RLA (HU) & $352 \pm 94$ & $340 \pm 89$ & 0.42 \\
\hline LUA (HU) & $341 \pm 84$ & $326 \pm 88$ & 0.27 \\
\hline LIA (HU) & $342 \pm 82$ & $327 \pm 89$ & 0.27 \\
\hline LLA (HU) & $357 \pm 88$ & $340 \pm 85$ & 0.21 \\
\hline $\begin{array}{l}\text { Subscapular and } \\
\text { paraspinal muscle }\end{array}$ & $44 \pm 9$ & $41 \pm 10$ & 0.12 \\
\hline Background noise & $10 \pm 4$ & $6 \pm 1$ & $<0.0001$ \\
\hline SNR & $43 \pm 20$ & $56 \pm 19$ & $<0.0001$ \\
\hline CNR & $38 \pm 18$ & $50 \pm 17$ & $<0.0001$ \\
\hline CTDIvol & $8 \pm 1.8$ & $0.59 \pm 0.003$ & $<0.0001$ \\
\hline DLP (mGy cm) & $282 \pm 64$ & $21 \pm 2$ & $<0.0001$ \\
\hline Effective dose (mSv) & $4.1 \pm 1.1$ & $0.3 \pm 0.03$ & $<0.0001$ \\
\hline $\operatorname{SSDE}(m G y)$ & $13.3 \pm 3.7$ & $0.74 \pm 0.14$ & $<0.0001$ \\
\hline
\end{tabular}

Pulmonary arteries represented the mean attenuation of all the arteries

$M P A$ main pulmonary artery, $R P A$ right pulmonary artery, $L P A$ left pulmonary artery, $R U A$ right upper lobe artery, $R M A$ right middle lobe artery, $R L A$ right lower lobe artery, $L U A$ left upper lobe artery, LIA lingular artery, $L L A$ left lower lobe artery

$(p<0.0001)$ for low $\mathrm{mA}$ MBIR-CTPA and routine FBPCTPA, respectively.

Quantitative image analysis

The low mA MBIR-CTPA yielded the same attenuation in each of the nine arteries of the lung, a lower noise and a better
SNR and CNR, despite a significant $\mathrm{x}$-ray dose reduction, when compared to classical FBP-CTPA (Table 2).

Qualitative image analysis

Table 3 summarizes the image quality of the low mA MBIRCTPA and of the routine FBP-CTPA protocol for each reader. The inter-reader agreements were excellent for both routine FBP-CTPA and low mA MBIR-CTPA, with kappa values of 0.921 and 0.865 , respectively. In the routine FBP-CTPA group, there was only one examination (for reader 1) which was considered as non-diagnostic. This was attributed to a suboptimal injection time. The non-diagnostic examinations in the low mA MBIR-CTPA groups ( $n=7$ for both readers) were mostly due to patients with a BMI greater than $30 \mathrm{~kg} / \mathrm{m}^{2}$ $(n=6)$ and due to suboptimal injection time in one case.

Figure 1 illustrates a typical case of routine FBP-CTPA and low mA MBIR-CTPA for patients with BMI less than $20 \mathrm{~kg} /$ $\mathrm{m}^{2}$, BMI between 20 and $30 \mathrm{~kg} / \mathrm{m}^{2}$, and BMI greater than $30 \mathrm{~kg} / \mathrm{m}^{2}$.

Figure 2 shows the presence of a non-occlusive PE in the right lower lobe artery on a low mA MBIR-CTPA, confirmed 3 days later by a routine FBP-CTPA.

\section{Discussion}

Our prospective study shows that low mA MBIR-CTPA allows for a significant $\mathrm{x}$-ray dose reduction of $93 \%$, while maintaining the same attenuation in each of the nine pulmonary arteries investigated. Moreover, as the noise was significantly lower when using MBIR, the SNR and CNR were significantly higher on the low mA MBIR-CTPA than on the routine FBP-CTPA protocol. In this paper, and despite the very low dose level achieved, we do not use the term "ultra-

Table 3 Subjective image quality of low $\mathrm{mA}$ MBIR-CTPA and routine FBP-CTPA for each reader

\begin{tabular}{|c|c|c|c|c|c|}
\hline & Reader 1 & & Reader 2 & & Kappa \\
\hline FBP-CTPA (subjective image quality) & $\mathrm{BMI}<30$ & $\mathrm{BMI}>30$ & $\mathrm{BMI}<30$ & $\mathrm{BMI}>30$ & 0.921 \\
\hline 1 & 0 & 0 & 0 & 0 & \\
\hline 2 & 0 & 0 & 0 & 0 & \\
\hline 3 & 1 & 0 & 0 & 0 & \\
\hline 4 & 0 & 2 & 3 & 1 & \\
\hline 5 & 63 & 16 & 61 & 17 & \\
\hline MBIR-CTPA (subjective image quality) & $\mathrm{BMI}<30$ & $\mathrm{BMI}>30$ & $\mathrm{BMI}<30$ & $\mathrm{BMI}>30$ & 0.865 \\
\hline 1 & 0 & 3 & 0 & 2 & \\
\hline 2 & 0 & 3 & 0 & 3 & \\
\hline 3 & 1 & 0 & 1 & 1 & \\
\hline 4 & 8 & 1 & 6 & 0 & \\
\hline 5 & 59 & 7 & 61 & 8 & \\
\hline
\end{tabular}


Fig. 1 Coronal reformatted thin slice CT ( $2 \mathrm{~mm}$ slice thickness) of routine FBP-CTPA (first row) and of low $\mathrm{MA}$ MBIR-CTPA (second row) of patients with a BMI less than $20 \mathrm{~kg} / \mathrm{m}^{2}$ (a, d), with a BMI between 20 and $30 \mathrm{~kg} / \mathrm{m}^{2}(\mathbf{b}, \mathbf{e})$ and with a BMI greater than $30 \mathrm{~kg} / \mathrm{m}^{2}$ (c, f). The representative images demonstrated an excellent image quality for all patients with a BMI less than $30 \mathrm{~kg} / \mathrm{m}^{2}$
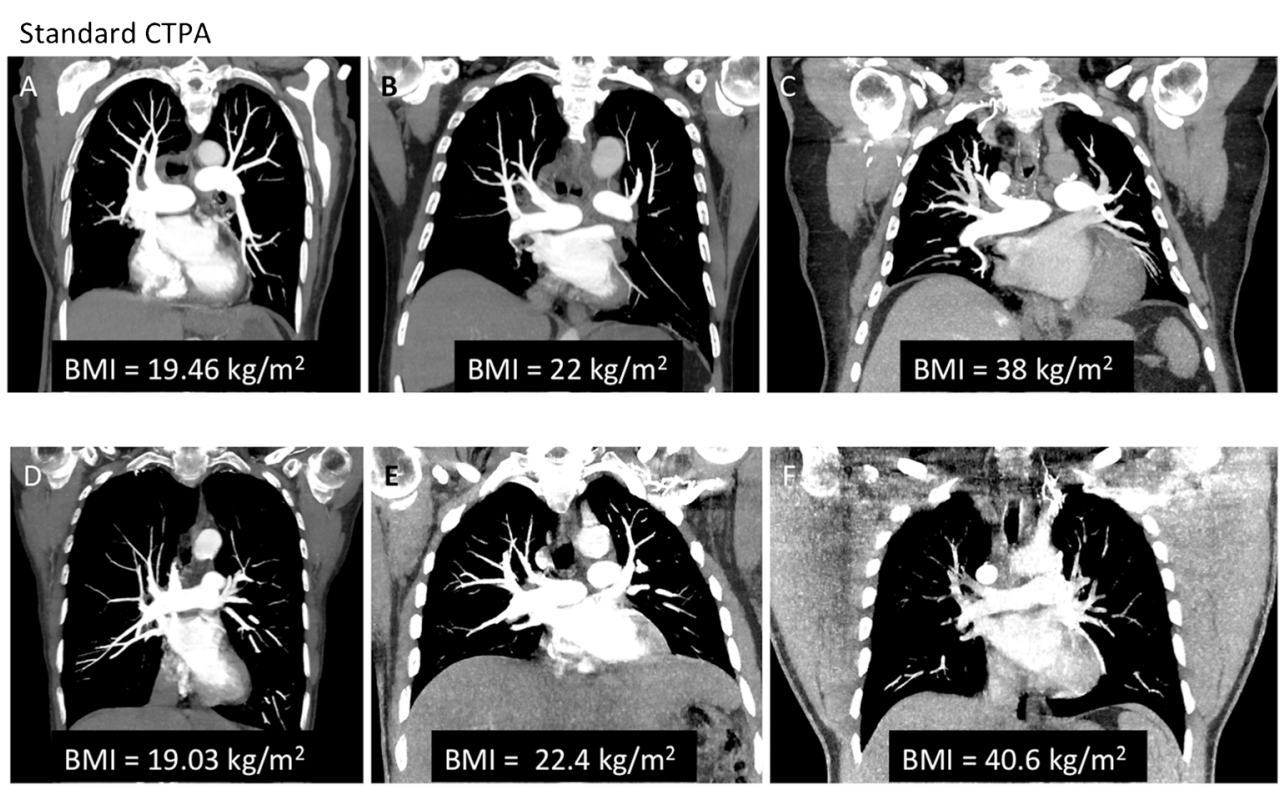

ULD-CTPA low dose CTPA" in accordance with the editorial by Bankier and Kressel [22], in which they state that the terms "low", "ultra-low" and "even super-extra-nano-low-dose" should not be used anymore. Instead, we indicate the DLP, CTDIvol, effective dose and SSDE for objective comparison with other studies.

High sensitivity (83-100\%) and high specificity (89$98 \%$ ) have been reported for routine CTPA for diagnosing pulmonary embolism (PE) [23-25]. However, because of the relatively low prevalence (9-35\%) of PE among patients evaluated by CTPA, one must be extremely cautious with the dose delivered to patients $[8,26,27]$, as it has been estimated that 5 fatalities per 100,000 persons per mSv could be expected [28].

Previous reports investigated the image quality of CTPA after dose reduction due to decrease $\mathrm{kVp}$ or $\mathrm{mA}$ or both $[5,29$, $30]$ or due to lowering of the $z$ coverage $[31,32]$. All these

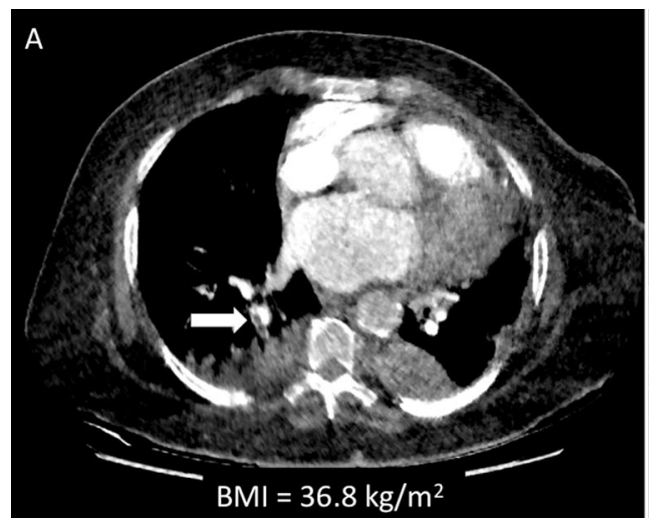

Fig. 2 Axial thin slice CT (0.625 mm slice thickness) demonstrating a pulmonary embolism (arrow) in the right lower lobe artery picked up on the low mA MBIR-CTPA (a), confirmed 3 days later (arrow) on a routine reports showed that PE could be diagnosed with a DLP varying from 40 to $110 \mathrm{mGy} \mathrm{cm}$. Particularly, it was shown that the use of $100 \mathrm{kVp}$ was possible, without significant loss of image quality [5]. Hence, $100 \mathrm{kVp}$ was used in this study for both the routine FBP-CTPA and low mA MBIR-CTPA. Our study uses a lower DLP of around $21 \mathrm{mGy} \mathrm{cm}$. This was made possible by the use of a second generation of iterative reconstruction, the so-called model-based iterative reconstruction. This type of reconstruction has already been applied to unenhanced chest imaging, where a dose of radiation close to the one delivered by a posteroanterior and lateral chest x-rays were used [16]. The MBIR algorithm makes fewer approximations than the FBP algorithm. MBIR takes into consideration a model of noise, a model of the object and a model of the optical properties of the CT. Taking into account all these models is computationally intense and around $40 \mathrm{~min}$ is needed for the reconstruction to be done. It is nowadays

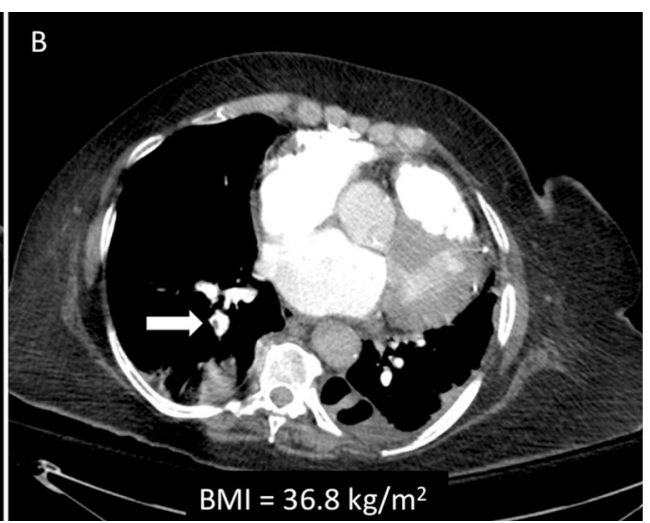

FBP-CTPA (b). Please note that the BMI of this patient was $36.8 \mathrm{~kg} / \mathrm{m}^{2}$, explaining the low image quality of the low $\mathrm{mA}$ MBIR-CTPA. Despite this, the PE was clearly identified on the images 
clearly a limitation, but the time needed for the reconstruction to be done should be significantly reduced with newer and faster calculators.

It has been reported that a diagnostic CTPA examination should have a contrast-enhanced blood attenuation of 200$250 \mathrm{HU}$ with a noise level below $22 \mathrm{HU}[28,33,34]$. Low $\mathrm{mA}$ MBIR-CTPA reached both criteria with a mean attenuation of $340 \pm 80 \mathrm{HU}$ and a background noise of $6 \pm 1$. The subjective image quality of all patients with a BMI less than $30 \mathrm{~kg} / \mathrm{m}^{2}$ was considered by both readers as diagnostic, except for one patient. The subjective image quality of all patients with a BMI greater than $30 \mathrm{~kg} / \mathrm{m}^{2}$ was graded as insufficient for diagnostic purpose (in six patients). This corresponds to $43 \%$ of potentially non-diagnostic CTs in this category of patients. The use of low mA MBIR-CTPA protocols in obese patients (i.e. a BMI greater than $30 \mathrm{~kg} / \mathrm{m}^{2}$ ) should then be strongly discouraged. Future studies should be undertaken to investigate how far the dose could be reduced in accordance with patient BMI.

\section{Study limitation}

Two different cohorts of patients were investigated and therefore a direct comparison in the same patients between low $\mathrm{mA}$ MBIR-CTPA and routine FBP-CTPA was not possible. It was difficult, from an ethical point of view, to inject each patient twice during the same imaging session, which is why two groups of patients have been compared.

There is only one PE in the low MA MBIR-CTPA protocols (Fig. 2), which is clearly not enough to draw conclusions of the clinical efficacy of this new low dose protocol. Nevertheless, the primary objective of this study was to establish the possibility of acquiring low $\mathrm{mA}$ MBIR-CTPA and to assess its image quality. Dose reduction was obtained using reduction of $\mathrm{mA}$ without reduction of $\mathrm{kVp}$, leaving the higher attenuation of iodine at low $\mathrm{kVp}$ unutilized. We were afraid of having too many artefacts in the shoulder region using low $\mathrm{kVp}$ and low $\mathrm{mA}$. This should be studied in the future.

Moreover, as the patients included in the low mA MBIRCTPA protocol were not suspected of having PE, the probability of finding a PE was low.

Since MBIR is one of the only fully iterative algorithms commercially available [12], it is unclear if our results are applicable to other iterative reconstruction algorithms.

\section{Conclusion}

Our prospective study showed that low mA MBIR-CTPA allows a significant dose reduction while improving SNR and CNR in the pulmonary vessels, as compared with routine FBP-CTPA. This first study was a necessary step to control image quality of low mA MBIR-CTPA. The results showed that low mA MBIR-CTPA can now be studied in the clinical context of suspected PE.

Acknowledgments Statistical support was provided by the Clinical Research Center, University of Geneva and Geneva University Hospitals (Delphine Courvoisier). The scientific guarantor of this publication is Xavier Montet. The authors of this manuscript declare no relationships with any companies whose products or services may be related to the subject matter of the article. The authors state that this work has not received any funding. One of the authors has significant statistical expertise. No complex statistical methods were necessary for this paper. Institutional review board approval was obtained. Written informed consent was obtained from all subjects (patients) in this study. Methodology: prospective, experimental, performed at one institution.

\section{References}

1. Furlan A, Aghayev A, Chang CC et al (2012) Short-term mortality in acute pulmonary embolism: clot burden and signs of right heart dysfunction at CT pulmonary angiography. Radiology 265:283-293

2. Sheh SH, Bellin E, Freeman KD et al (2012) Pulmonary embolism diagnosis and mortality with pulmonary $\mathrm{CT}$ angiography versus ventilation-perfusion scintigraphy: evidence of overdiagnosis with CT? AJR Am J Roentgenol 198:1340-1345

3. Remy-Jardin M, Pistolesi M, Goodman LR et al (2007) Management of suspected acute pulmonary embolism in the era of CT angiography: a statement from the Fleischner Society. Radiology 245:315-329

4. Mettler FA Jr, Huda W, Yoshizumi TT et al (2008) Effective doses in radiology and diagnostic nuclear medicine: a catalog. Radiology 248: 254-263

5. Heyer CM, Mohr PS, Lemburg SP et al (2007) Image quality and radiation exposure at pulmonary CT angiography with 100 - or 120 $\mathrm{kVp}$ protocol: prospective randomized study. Radiology 245:577-583

6. Zamboni GA, Guariglia S, Bonfante A et al (2012) Low voltage CTPA for patients with suspected pulmonary embolism. Eur J Radiol 81:e580-e584

7. Henes FO, Groth M, Begemann PG et al (2012) Impact of tube current-time and tube voltage reduction in 64-detector-row computed tomography pulmonary angiography for pulmonary embolism in a porcine model. J Thorac Imaging 27:113-120

8. Tack D, De Maertelaer V, Petit W et al (2005) Multi-detector row CT pulmonary angiography: comparison of standard-dose and simulated low-dose techniques. Radiology 236:318-325

9. Kalra MK, Woisetschlager M, Dahlstrom N et al (2013) Sinogramaffirmed iterative reconstruction of low-dose chest CT: effect on image quality and radiation dose. AJR Am J Roentgenol 201: W235-W244

10. Li Q, Yu H, Zhang L et al (2013) Combining low tube voltage and iterative reconstruction for contrast-enhanced CT imaging of the chest-initial clinical experience. Clin Radiol 68:e249-e253

11. Vardhanabhuti V, Loader RJ, Mitchell GR et al (2013) Image quality assessment of standard- and low-dose chest CT using filtered back projection, adaptive statistical iterative reconstruction, and novel model-based iterative reconstruction algorithms. AJR Am J Roentgenol 200:545-552

12. Willemink MJ, de Jong PA, Leiner T et al (2013) Iterative reconstruction techniques for computed tomography Part 1: technical principles. Eur Radiol 23:1623-1631

13. Deak Z, Grimm JM, Treitl M et al (2013) Filtered back projection, adaptive statistical iterative reconstruction, and a model-based iterative reconstruction in abdominal CT: an experimental clinical study. Radiology 266:197-206 
14. Katsura M, Matsuda I, Akahane M et al (2013) Model-based iterative reconstruction technique for ultralow-dose chest CT: comparison of pulmonary nodule detectability with the adaptive statistical iterative reconstruction technique. Invest Radiol 48:206-212

15. Pickhardt PJ, Lubner MG, Kim DH et al (2012) Abdominal CT with model-based iterative reconstruction (MBIR): initial results of a prospective trial comparing ultralow-dose with standard-dose imaging. AJR Am J Roentgenol 199:1266-1274

16. Neroladaki A, Botsikas D, Boudabbous S et al (2013) Computed tomography of the chest with model-based iterative reconstruction using a radiation exposure similar to chest $\mathrm{x}$-ray examination: preliminary observations. Eur Radiol 23:360-366

17. Christensen E (2007) Methodology of superiority vs. equivalence trials and non-inferiority trials. J Hepatol 46:947-954

18. Kanal KM, Stewart BK, Kolokythas O et al (2007) Impact of operator-selected image noise index and reconstruction slice thickness on patient radiation dose in 64-MDCT. AJR Am J Roentgenol 189:219-225

19. European Commission (1999) European guidelines on quality criteria for computed tomography. Report EUR 16262. European Commission, Brussels

20. Brady SL, Kaufman RA (2012) Investigation of American Association of Physicists in Medicine Report 204 size-specific dose estimates for pediatric CT implementation. Radiology 265:832-840

21. Brink JA, Morin RL (2012) Size-specific dose estimation for CT: how should it be used and what does it mean? Radiology 265:666-668

22. Bankier AA, Kressel HY (2012) Through the looking glass revisited: the need for more meaning and less drama in the reporting of dose and dose reduction in CT. Radiology 265:4-8

23. Coche E, Verschuren F, Keyeux A et al (2003) Diagnosis of acute pulmonary embolism in outpatients: comparison of thin-collimation multi-detector row spiral CT and planar ventilation-perfusion scintigraphy. Radiology 229:757-765

24. Stein PD, Fowler SE, Goodman LR et al (2006) Multidetector computed tomography for acute pulmonary embolism. N Engl J Med 354:2317-2327
25. Winer-Muram HT, Rydberg J, Johnson MS et al (2004) Suspected acute pulmonary embolism: evaluation with multi-detector row CT versus digital subtraction pulmonary arteriography. Radiology 233: 806-815

26. Diederich S (2003) Radiation dose in helical CT for detection of pulmonary embolism. Eur Radiol 13:1491-1493

27. Wells PS, Anderson DR, Rodger M et al (2001) Excluding pulmonary embolism at the bedside without diagnostic imaging: management of patients with suspected pulmonary embolism presenting to the emergency department by using a simple clinical model and ddimer. Ann Intern Med 135:98-107

28. Mayo J, Thakur Y (2013) Pulmonary CT angiography as first-line imaging for PE: image quality and radiation dose considerations. AJR Am J Roentgenol 200:522-528

29. Litmanovich D, Boiselle PM, Bankier AA et al (2009) Dose reduction in computed tomographic angiography of pregnant patients with suspected acute pulmonary embolism. J Comput Assist Tomogr 33: 961-966

30. MacKenzie JD, Nazario-Larrieu J, Cai T et al (2007) Reduced-dose CT: effect on reader evaluation in detection of pulmonary embolism. AJR Am J Roentgenol 189:1371-1379

31. Kallen JA, Coughlin BF, O'Loughlin MT et al (2010) Reduced z-axis coverage multidetector $\mathrm{CT}$ angiography for suspected acute pulmonary embolism could decrease dose and maintain diagnostic accuracy. Emerg Radiol 17:31-35

32. Martillotti J, Silva N, Chhabra J et al (2013) Dose of reduced z-axis length of computed tomography angiography (CTA) of the chest for pulmonary embolism using 64-detector rows and adaptive iterative reconstruction techniques. Emerg Radiol 20:3944

33. Jones SE, Wittram C (2005) The indeterminate CT pulmonary angiogram: imaging characteristics and patient clinical outcome. Radiology 237:329-337

34. Ramadan SU, Kosar P, Sonmez I et al (2010) Optimisation of contrast medium volume and injection-related factors in CT pulmonary angiography: 64-slice CT study. Eur Radiol 20:2100-2107 